\title{
NEW PUBLICATIONS
}

\section{PART I. PURE MATHEMATICS}

Allen (E. S.). Six-place tables. McGraw-Hill, New York, 1935. 23+175 pp. BachmanN (F.). Untersuchungen zur Grundlegung der Arithmetik mit besonderer Beziehung auf Dedekind, Frege, und Russell. (Forschungen zur Logistik und zur Grundlegung der exakten Wissenschaften, Heft 1.) Leipzig, Meiner, 1934. $78 \mathrm{pp}$.

BAER (R.). Automorphismen von Erweiterungsgruppen. (Actualités Scientifiques et Industrielles, No. 205: Exposés mathématiques, publiés à la mémoire de J. Herbrand, X.) Paris, Hermann, 1935. 22 pp.

Balliccioni (A.). See Couderc (P.).

Boll (M.). See Hahn (H.).

CARATHÉODORY (C.). Variationsrechnung und partielle Differentialgleichungen erster Ordnung. Leipzig und Berlin, Teubner, 1935. 11+407 pp.

Couderc (P.) et Balliccioni (A.). Premier livre du tétraèdre à l'usage des élèves de première, de mathématiques des candidats aus grandes écoles et à l'agrégation. Préface de M. H. Villat. Paris, Gauthier-Villars, 1935. $8+199 \mathrm{pp}$.

Cox (S. H. J.). A concise algebra. London, Rivingtons, 1935. 8+164+13 pp.

Deuring (M.). Algebren. (Ergebnisse der Mathematik und ihrer Grenzgebiete, herausgegeben von der Schriftleitung des "Zentralblatt für Mathematik," Band 4, Teil 1.) Berlin, Springer, 1935. 5+143 pp.

Ebner (F.) und Roth (L.). Technische Mathematik. Differential- und Integralrechnung. Leipzig, Teubner, 1935. 167 pp.

HahN (H.). Logique, mathématiques et connaissance de la réalité. Traduction du E. Vouillemin, introduction de M. Boll. (Actualités Scientifiques et Industrielles, No. 226.) Paris, Hermann, 1935. 53 pp.

Hegel (G. W. F.). Sämtliche Werke. Herausgegeben von G. Lasson. Band 4: Wissenschaft der Logik. (Die Philosophische Bibliothek, Band 57.) 2te Auflage. Leipzig, Meiner, 1934. $511 \mathrm{pp}$.

Heisenberg (W.). Wandlungen in den Grundlagen der Naturwissenschaft: zwei Vorträge. Leipzig, Hirzel, $1935.45 \mathrm{pp}$.

Herbrand (J.). See Baer (R.), von Neumann (J.), Weil (A.).

KäHLER (E.). Einführung in die Theorie der Systeme von Differentialgleichungen. (Hamburger Mathematische Einzelschriften, Heft 16.) Leipzig und Berlin, Teubner, 1934. $78 \mathrm{pp}$.

LAGALly (M.). Vorlesungen über Vektorrechnung. 2te verbesserte Auflage. Leipzig, Akademische Verlagsgesellschaft, 1934.

Lasson (G.). See Hegel (G. W. F.).

LeCAT (M.). Erreurs de mathématiciens: des origines à nos jours. Brussels, Castaigne, 1935.

von NeumanN (J.). Charakterisierung des Spektrums eines Integraloperators. (Actualités Scientifiques et Industrielles, No. 229: Exposés mathéma- 
tiques, publiés à la mémoire de J. Herbrand, XIII.) Paris, Hermann, 1935. $20 \mathrm{pp}$.

Rey (A.). Les mathématiques en Grèce au milieu de $\mathrm{V}^{\mathrm{e}}$ siècle. (Actualités Scientifiques et Industrielles, No. 207.) Paris, Hermann, 1935. 92 pp.

Roth (L.). See EBNER (F.).

Rothe (R.). Höhere Mathematik. Teil III: Raumkurven und Flächen Linienintegrale und mehrfache Integrale gewöhnliche und partielle Differentialgleichungen nebst Anwendungen. (Teubners Mathematische Leitfaden, Band 23.) Leipzig und Berlin, Teubner, 1935. 9+238 pp.

Sansone (G.). See Vitali (G.).

Schilling (F.). Die Pseudosphäre und die nichteuklidische Geometrie. I und II Teil in einem Band. Leipzig und Berlin, Teubner, 1935. 4+215 pp.

Schmidt (K.). Das geometrische Problem der Kreishalbierung. Leipzig und Berlin, Teubner, 1935. $26 \mathrm{pp}$.

Schouten (J. A.) und Struik (D. J.). Einführung in die neueren Methoden der Differentialgeometrie. 2te vollständig umgearbeitete Auflage. Band I: Algebra und Übertragungslehre von J. A. Schouten. Groningen-Batavia, Noordhoff, 1935. 12+202 pp.

Struik (D. J.). See Schouten (J. A.).

Villat (M. H.). See Couderc (P.).

Vitali (G.) e SAnsone (G.). Moderna teoria delle funzioni di variabile reale. Parte prima. (Monografie di Matematica Applicata.) Bologna, Zanichelli, 1935. 183 pp.

Vouillemin (E.). See Hahn (H.).

WARRAIN (F.). Essai sur les principes des algorithmes primitifs: addition, soustraction, multiplication, division, puissances, racines. (Institut Général Psychologique, reconnu d'utilité publique, Mémoires, No. 6; Commission International de Détermination Mathématique des Phénomènes Psycho- et Socio-biologiques, Mémoires, No. 1.) Paris, Hermann, 1934. $147 \mathrm{pp}$.

WEIL (A.). Arithmétique et géométrie sur les variétés algébriques. (Actualités Scientifiques et Industrielles, No. 206: Exposés mathématiques, publiés à la mémoire de J. Herbrand, XI.) Paris, Hermann, 1935. 16 pp.

Witting (A.). Repetitorium und Aufgabensammlung zur Differentialrechnung. (Sammlung Göschen, Band 146.) Berlin, de Gruyter, 1935. 136 pp.

Zygmund (A.). Trigonometrical series. (Monografje Matematyczne, Tome 5.) Warsaw, Seminarjum Matematyczne Uniwersytetu Warszawskiego, 1935. $4+332 \mathrm{pp}$.

\section{PART II. APPLIED MATHEMATICS}

Ameline (M.). La roulette et son zéro (Les possibilités, l'écart ou gainperte. Les jeux avec retard). Bourges, Librairie Desquand, 1934. 83 pp.

Defay (R.). Étude thermodynamique de la tension superficielle. Préface de Th. de Donder. Paris, Gauthier-Villars, 1934. 11+372 pp.

De Donder (T.). See Defay (R.).

van DEN Dungen (F. H.). Acoustique des salles. (Institut Belge de Recherches Radioscientifiques, Tome 2.) Paris, Gauthier-Villars, 1934. 7+118 pp. 
Eddington (A.). New pathways in science. (Messenger Lectures, 1934.) Cambridge, University Press, $1935.10+333$ pp.

ERRERA (J.). Le moment électrique en chimie et en physique; généralités et méthodes. (Actualités Scientifiques et Industrielles, No. 220.) Paris, Hermann, 1935. 47 pp.

_- Le moment électrique en chimie et en physique; moment électrique et structure moléculaire. (Actualités Scientifiques et Industrielles, No. 221.) Paris, Hermann, 1935. 60 pp.

Eucken (A.). See Fuchs (O.).

FACx (L.) et Guieysse (G.). Solutions de problèmes et compléments d'astronomie. Paris, Mercure universel, 1935. $154 \mathrm{pp}$.

Fric (R.). See Thomson (G. P.), Thomson (J. J.).

Fuchs (O.) und Wolf (K. L.). Dielektrische Polarisation. (Hand- und Jahrbuch der chemischen Physik, herausgegeben von A. Eucken und K. L. Wolf, Band 6: Elektrizität und Materie, Abschnitt 1B.) Leipzig, Akademische Verlagsgesellschaft, $1935.10+232+12 \mathrm{pp}$.

Gebelein (H.). Turbulenz: Physikalische Statistik und Hydrodynamik. Berlin, Springer, 1935. 8+177 pp.

Glover (J. W.). See Menge (W. O.).

Guieysse (G.). See Facy (L.).

Heymann (A.). Über thermomechanische Gasströme. Dresden, Risse Verlag, $1934.42 \mathrm{pp}$.

Hicks (W. M.). The structure of spectral terms. London, Methuen, 1935. $11+209$ pp.

Holzer (W.) und Weissenberg (E.). Grundriss der Kurzwellentherapie: Physik, Technik, Indikationen; Einführung in die physikalisch-technischen und medizinischen Grundlagen der Anwendung kurzer elektrischer Wellen für Ärzte und Biologen. Physik und Technik, von W. Holzer; Medizinische Anwendung, von E. Weissenberg. Vienna, Maudrich, 1935. $6+189 \mathrm{pp}$.

Joffé (A. F.). Semi-conducteurs électroniques. (Actualités Scientifiques et Industrielles, No. 202.) Paris, Hermann, 1935. $91 \mathrm{pp.}$

Johnston (W. H.). See SchröDinger (E.).

Karlson (P.). Du und die Natur: eine moderne Physik für Jedermann. Berlin, Verlag Ullstein, 1934. 355 pp.

KNOLl (M.), OllendorfF (F.), und Rompe (R.). Gasentladungs-Tabellen: Tabellen, Formeln und Kurven zur Physik und Technik der Elektronen und Ionen. Unter Mitarbeit von A. Roggendorf. Berlin, Springer, 1935. $10+171 \mathrm{pp}$.

LANGLOIS-BERTHELOT (R.). Les machines asynchrones à champst ournants, à bagues et à collecteur. Paris, Dunod, $1934.44+276$ pp.

LoHse (-.). Tafeln für numerisches Rechnen mit Maschinen. 2te Auflage, neubearbeitet von P.V. Neugebauer. Leipzig, Engelmann, 1935. 5+113 pp.

Menge (W. O.) and Glover (J. W.). An introduction to the mathematics of life insurance. New York, Macmillan, 1935. 9+190 pp.

MILNE (E. A.). Relativity, gravitation, and world-structure. (International Series of Monographs on Physics.) Oxford, Clarendon Press; London, Oxford University Press, 1935. 10+365 pp. 
Murphy (J.). See SchröDinger (E.).

Neugebauer (P. V.). See Lohse (-.).

OllendorfF (F.). See Knoll (M.).

Paterson (C. C.). The electron liberated; its industrial consequences. (Lectures to a Group of London Schools.) London, British Science Guild, 1935.

Prange (G.). Die allgemeinen Integrationsmethoden der analytischen $\mathrm{Me}$ chanik. (Encyklopädie der mathematischen Wissenschaften mit Einschluss ihrer Anwendungen, Band IV, Heft 4.) Leipzig, Teubner, 1935. $13+299$ pp.

Rapanastassiou (C. E.). Les théories sur la nature de la lumière, de Descartes à nos jours et l'évolution de la théorie physique. Paris, Jouve, 1935. $162 \mathrm{pp}$.

RogGendorf (A.). See KNoll (M.).

Rompe (R.). See KNoll (M.).

Scheminsky (F.). Die Welt des Schalles. Graz, "Das Bergland-Buch," 1935. $742 \mathrm{pp}$.

SchröDINGer (E.). Science and the human temperament. Translated by Dr. James Murphy and W. H. Johnston. New York, Norton, 1935. 24+192 pp.

Semenoff (N.). Chemical kinetics and chain reactions. (International Series of Monographs on Physics.) Oxford, Clarendon Press; London, Oxford University Press, $1935.12+480 \mathrm{pp}$.

Shearcroft (W. F. F.). Elementary light. Part 1. (Dent's Modern Science Series.) London and Toronto, Dent, 1935. $89 \mathrm{pp}$.

Smith (S. P.), edited by. Problems in electrical engineering with answers. 2d edition, revised and enlarged. London, Constable, 1935. 14+210 pp.

Thomson (G. P.). Diffraction des rayons cathodiques. Traduit de l'anglais par R. Fric. (Actualités Scientifiques et Industrielles, No. 212.) Paris, Hermann, 1935. $56 \mathrm{pp}$.

Thomson (J. J.). Au dela de l'électron. Traduit de l'anglais par R. Fric. (Actualités Scientifiques et Industrielles, No. 211.) Paris, Hermann, 1935. $30 \mathrm{pp}$.

Thол (N.). L'électrolyse et la polarisation électrolytique. (Actualités Scientifiques et Industrielles, No. 166.) Paris, Hermann, 1934. 33 pp.

WeCKering (R.). Stéréophysique. Nouvelles théories sur la constitution de la matière et l'origine des rayonnements. Structure dans l'espace des édifices atomiques et moléculaires. Processus physiques à l'origine des spectres de raies. Paris, Dunod, 1935. $662 \mathrm{pp}$.

Weissenberg (E.). See Holzer (W.).

Wolf (K. L.). See Fuchs (O.). 